\title{
New Applications of the Calculus of Variations in the Large to Nonlinear Elasticity
}

\author{
Melvyn S. Berger* \\ Institute for Advanced Study. Princeton University, Princeton, New Jersey 08540, USA
}

Received July 1, 1973

\begin{abstract}
Global methods of the calculus of variations and the infinite dimensional critical point theories of Morse and Ljusternik are applied to investigate the structure of the equilibrium states of thin flexible elastic plates under general body forces. The arguments used are equally applicable to broad classes of physical systems governed by nonlinear elliptic partial differential equations.
\end{abstract}

In this paper we take up the problem of determining the global structure of the equilibrium states of a thin elastic plate (of arbitrary shape) under the action of quite general body forces. We assume throughout that the deformations are governed by the well-known (nonlinear) von Karman equations, and we use a number of new and far-reaching ideas in the calculus of variations in the large to study the global structure of the solutions of these equations. Actually the von Karman equations have a particularly interesting Hamiltonian structure, so that one of the main purposes of this paper is the determination of the theoretical implications of this structure.

In papers [1] and [2], the author (together with Fife) applied some results from the calculus of variations in the large to study the buckling of a thin elastic plate under rather general edge conditions. In this paper we take up the general problem of studying the combined bucklingbending problem. Previous determinations of the structure of the resulting equilibrium states have been primarily restricted to rather local considerations (for example [3]). By studying the qualitative features of the nonlinear operator associated with the von Karman equations, we are able to obtain global results on the structure of the equilibrium states that perhaps explain some of the complicated phenomena observed experimentally.

Our paper is organized as follows: In Section I we formulate the mathematical problem to be considered and review the basic information concerning this problem already established in our earlier papers. In Section II we investigate those qualitative features of the von Karman

\footnotetext{
* Research partially supported by AFOSR grant 73-2437.
} 
equations that will be used in the sequel. Section III is devoted to applying these qualitative features to ascertain characteristics of the global structure of the equilibrium states.

The von Karman equations have particularly beautiful mathematical properties that make them well suited for the application of modern critical point theory. Not only do these equations always admit a "stable" solution under very general body forces, but one can give rather interesting variational characterizations of other "unstable" solutions of the equations. It can be conjectured that the various solutions found here lie on continuous curves (in function space), and the sharp exchange of stability observed in elastic deformations is caused by the transition of absolute minima among these curves. In any case, it is clear that the qualitative results obtained here are fundamental for the mathematical understanding of the structure of stationary states of elastic deformations under general stress-strain laws.

Hopefully, the investigations of the present paper will also be of interest in contemporary problems concerning symmetry breakdown and dynamic instability of infinite dimensional (nonlinear) Hamiltonian systems. Indeed, the present paper shows that such systems (in contrast to large amplitude motions of viscous incompressible fluids) can be treated globally by the methods of the calculus of variations in the large.

\section{Section I. The Mathematical Formulation of the Problem}

The physical problem to be discussed can be described as follows: a flat, thin elastic plate of arbitrary shape is subjected to forces along its edge as well as forces acting normal to the plane of the plate. The problem is to determine the resulting equilibrium states of the deformed plate, assuming the plate is subjected to general edge conditions as described in [2]. If the plate is represented by a bounded region $\Omega$ in the plane, the resulting von Karman equations governing the equilibrium states can be written in the form

$$
\begin{aligned}
\Delta^{2} f & =-[\omega, \omega] \\
\Delta^{2} \omega & =\lambda\left[F_{0}, \omega\right]+[f, \omega]+p,
\end{aligned}
$$

where $\Delta^{2}$ is the biharmonic operator and the quadratic form $[f, g]$ $=f_{x x} g_{y y}+f_{y y} g_{x x}-2 f_{x y} g_{x y}$. The meaning of the variables $f, \omega, F_{0}, \lambda$ and $p$ is easily explained. $\omega(x, y)$ represents the deflection of the plate from its flat unstressed position, $f+\lambda F_{0}$ is the Airy stress function in the plate, whereas $\lambda F_{0}$ is a given function representing the stress function in the undeflected plate with $p=0$. Finally $p$ represents the given force acting normal to the plane of the plate. Of course the partial 
differential equations $(1 \mathrm{a}, \mathrm{b})$ must be supplemented with boundary conditions representing the edge conditions for the problem. To simply the presentation here, we suppose these conditions are represented by the equations

$$
\begin{gathered}
\omega=\omega_{x}=\omega_{y}=0 \\
f=f_{x}=f_{y}=0
\end{gathered} \text { on } \quad \partial \Omega .
$$

All the results presented, however, are valid under the more general edge conditions of [2]. This follows directly from the theorems of [2], since only qualitative properties of the Eqs. $(1 \mathrm{a}-\mathrm{d})$ are used in the sequel. By a solution to system $(1 \mathrm{a}-\mathrm{d})$ we mean a pair $(f, \omega)$ which satisfy the equations in $\Omega$ and at all smooth portions of $\partial \Omega$.

The basic facts from our previous work, to be used here, are summarized in the following

Lemma $\mathbf{A}([1,2])$. The solutions $(\omega, f)$ of system $(1 \mathrm{a}-\mathrm{d})$ are identical with the solutions of the following operator equation defined on the Hilbert space $H \equiv \stackrel{\circ}{W}_{2,2}(\Omega)$

$$
u+C u-\lambda L u=g \text { with } \omega=u \text { and } f=-\frac{1}{2} C(u, u),
$$

where the operators $L u, C(u, v)$, and $C u$ are defined implicitly by the formulae

$$
\begin{aligned}
(L u, \eta) & =\int_{\Omega}\left[F_{0}, \omega\right] \eta \\
(C(u, v), \eta) & =\int_{\Omega}[u, v] \eta \quad \text { and } C u=C(C(u, u), u) \\
(g, \eta) & =\int_{\Omega} p \eta .
\end{aligned}
$$

Furthermore, the operator $L$ is a self-adjoint and compact mapping of $H$ into itself. The operator $C$ is a real analytic, completely continuous, gradient operator mapping $H$ into itself, with the additional properties:

(i) $\mathrm{Cu}$ is homogeneous of degree 3;

(ii) $(C u, u)=\|C(u, u)\|^{2} \geqq 0$, and equals zero if and only if $u=0$; and

(iii) the form $(C(u, v), w)$ is symmetric in $u, v$ and $w \in H$.

Lemma B. The solutions of the system $(1 \mathrm{a}-\mathrm{d})$ are identical with the critical points of the functional

$$
\mathscr{I}_{\lambda}(u)=\|u\|^{2}+\frac{1}{2}(C u, u)-\lambda(L u, u)-2(g, u) .
$$

Proof. The result is an immediate consequence of Theorem 2.3 of [1].

\section{Section II. Special Properties of the von Karman Equations}

In this section we shall study some interesting special properties of the operator $A_{\lambda} u=u+C u-\lambda L u$ defined on $H$ for fixed real $\lambda$. In order to describe the most important of these, we recall the notion of a proper 
mapping $g$ between two metric spaces $X$ and $Y$ : The mapping $g$ is said to be proper if the inverse image of a compact set in $Y$ is compact in $X$.

Theorem 1. For any fixed $\lambda \in(-\infty, \infty)$, the mapping $A_{\lambda}(u)=u+C u$ $-\lambda L u$, as defined in Lemma $A$, is a proper mapping of the Hilbert space $H$ into itself.

Proof. Let $\left\{g_{n}\right\}$ be any sequence in the range of $A_{\lambda}$, so that $A_{\lambda}\left(u_{n}\right)=g_{n}$ for some $u_{n} \in H$. It suffices to show that whenever $\left\{g_{n}\right\}$ converges to $g$, some subsequence of $\left\{u_{n}\right\}$ converges in $H$. To establish this fact we take the inner product of the equation $A_{\lambda} u_{n}=g_{n}$ with $u_{n}$ and write

$$
\begin{aligned}
\left(g_{n}, u_{n}\right)=\left(A_{\lambda} u_{n}, u_{n}\right) & =\left(u_{n}, u_{n}\right)-\lambda\left(L u_{n}, u_{n}\right)+\left(C u_{n}, u_{n}\right) \\
& =\left(u_{n}, u_{n}\right)-\lambda\left(C\left(F_{0}, u_{n}\right), u_{n}\right)+\left(C u_{n}, u_{n}\right) \\
& =\left(u_{n}, u_{n}\right)-\lambda\left(C\left(u_{n}, u_{n}\right), F_{0}\right)+\left\|C\left(u_{n}, u_{n}\right)\right\|^{2} .
\end{aligned}
$$

Thus for any $\varepsilon>0$ and any finite fixed $\lambda$

$$
\left(g_{n}, u_{n}\right) \geqq\left\|u_{n}\right\|^{2}-|\lambda| \varepsilon\left\|C\left(u_{n}, u_{n}\right)\right\|^{2}-(|\lambda| / \varepsilon)\left\|F_{0}\right\|^{2}+\left\|C\left(u_{n}, u_{n}\right)\right\|^{2} .
$$

Consequently setting $\varepsilon=1 /|\lambda|$ and using the Cauchy-Schwarz inequality, we find $\lambda^{2}\left\|F_{0}\right\|^{2}+\left\|g_{n}\right\| \geqq\left\|u_{n}\right\|$. Since $\left\|g_{n}\right\|$ is uniformly bounded, $\left\|u_{n}\right\|$ is also uniformly bounded, so that $\left\{u_{n}\right\}$ has a weakly convergent subsequence $\left\{u_{n_{j}}\right\}$ with

$$
u_{n_{j}}+C u_{n_{j}}-\lambda L u_{n_{j}}=g_{n_{j}} .
$$

Finally the complete continuity of $C$ and $L$ obtained in Lemma A, implies that the sequence $\left\{C u_{n_{j}}-L u_{n}\right\}$ converges strongly in $H$. Hence (6) implies that the sequence $\left\{u_{n_{j}}\right\}$ itself converges strongly in $H$. Thus the operator $A_{\lambda}$ is a proper mapping.

Corollary. Any solution of the Eq. (2) satisfies the a priori estimate

$$
\|\omega\| \leqq \frac{1}{2}\|g\|+\sqrt{\lambda^{2}\left\|F_{0}\right\|^{2}+\frac{1}{4}\|g\|^{2}} .
$$

Proof. We argue as in the proof of Theorem 1. Suppose $\omega-\lambda L \omega$ $+C \omega=g$. Then taking the inner product with $\omega$ and using the CauchySchwarz inequality, we find

$$
\|\omega\|^{2}-|\lambda|\left|\left(C(\omega, \omega), F_{0}\right)\right|+\|C(\omega, \omega)\|^{2} \leqq\|g\|\|\omega\| .
$$

Thus for any $\varepsilon>0$,

$$
\|\omega\|^{2}-|\lambda| \varepsilon\|C(\omega, \omega)\|^{2}-(|\lambda| / \varepsilon)\left\|F_{0}\right\|^{2}+\|C(\omega, \omega)\|^{2} \leqq\|g\|\|\omega\| .
$$

Choosing $\varepsilon=1 /|\lambda|$, we find

$$
\|\omega\|^{2} \leqq\|g\|\|\omega\|+\lambda^{2}\left\|F_{0}\right\|^{2} .
$$

Consequently the estimate (7) holds. 
Theorem 2. For fixed real $\lambda$, the operator $A_{\lambda} u$ is a Fredholm mapping of index zero; i.e. for each $u \in H$, the mapping $A_{\lambda}(u)$ has a Frechet derivative $A_{\lambda}{ }^{\prime}(u)$, and the linear operator $A_{\lambda}{ }^{\prime}(u)$ is a bounded linear Fredholm mapping of index zero.

Proof. By Lemma A, the operator $A_{\lambda}(u)$ is real analytic and consequently differentiable of all orders. On the other hand, by [4], the Frechet derivative of a completely continuous mapping is again completely continuous. Thus $A_{\lambda}{ }^{\prime}(u)$ exists for all $\lambda$ and can be represented as a compact self-adjoint perturbation of the identity. By [5], $A_{\lambda}{ }^{\prime}(u)$ is thus a linear bounded Fredholm mapping of index zero.

Theorem 3. For any fixed real $\lambda$ and any fixed $g \in H$, the functional defined by (3),

$$
\mathscr{I}_{\lambda}(u)=\|u\|^{2}+\frac{1}{2}(C u, u)-\lambda(L u, u)-2(g, u),
$$

is coercive (i.e. $\mathscr{I}_{\lambda}(u) \rightarrow \infty$ as $\|u\| \rightarrow \infty$ ). Therefore $\mathscr{I}_{\lambda}(u)$ is bounded below on $H$.

Proof. The proof proceeds almost exactly as in Theorem 1. Let $\left\{u_{n}\right\}$ be a sequence in $H$ with $\left\|u_{n}\right\| \rightarrow \infty$. Then, as in (4)-(5), for any $\varepsilon>0$,

$$
\begin{aligned}
\mathscr{I}_{\lambda}\left(u_{n}\right) & \geqq\left\|u_{n}\right\|^{2}+\left\|C\left(u_{n}, u_{n}\right)\right\|^{2}-|\lambda| \varepsilon\left\|C\left(u_{n}, u_{n}\right)\right\|^{2}-(|\lambda| / \varepsilon)\left\|F_{0}\right\|^{2}-2\left(g, u_{n}\right) \\
& \left.\geqq\left\|u_{n}\right\|^{2}-\lambda^{2}\left\|F_{0}\right\|^{2}-2\left\|g_{n}\right\|\left\|u_{n}\right\| \quad \text { (for } \quad \varepsilon=1 /|\lambda|\right) .
\end{aligned}
$$

Therefore $\mathscr{I}_{\lambda}\left(u_{n}\right) \rightarrow \infty$ as $\left\|u_{n}\right\| \rightarrow \infty$.

The most important consequences of the properties expressed in Theorems $1-3$ are:

Theorem 4. Let $S$ be the set $\left\{u \mid u \in H, A_{\lambda}{ }^{\prime}(u)\right.$ is not an invertible linear operator $\}$, i.e. the bifurcation set. Then for fixed real $\lambda$ :

(i) $A_{\lambda}(S)$ is a closed subset of $H$ which is nowhere dense in $H$;

(ii) For each $g \in\left(H-A_{\lambda}(S)\right)$, the equation $A_{\lambda}(u)=g$ has at most $a$ finite number of solutions;

(iii) The number of solutions of $A_{\lambda}(u)=g$ for $g$ in any component of $H-A_{\lambda}(S)$ is constant;

(iv) The functional $\mathscr{I}_{\lambda}(u)$ satisfies the Palais-Smale Condition $C$ on the Hilbert space $H$ (i.e. if $\mathscr{I}_{\lambda}\left(u_{n}\right) \leqq M$ on $H$, and $\mathscr{I}_{\lambda}\left(u_{n}\right) \rightarrow 0$, then $\left\{u_{n}\right\}$ has a convergent subsequence).

(v) The functional $\mathscr{I}_{\lambda}(u)$ satisfies the Morse inequalities [6, p. 338] for $g \in\left(H-A_{\lambda}(S)\right)$.

Proof. All the conclusions [except (iii)] follow from quite wellknown results in the mathematical literature.

(i) follows from the paper [11] of Smale and from Theorem 2.

(ii) follows from the properness of the mapping $A_{\lambda}(u)$. Indeed, if the equation $A_{\lambda}(u)=g$ has an infinite number of solutions $u_{1}, u_{2}, \ldots$ for $g \in\left(H-A_{\lambda}(S)\right)$, then the elements $u_{n}$ must have a limit point $\bar{u}$ that also satisfies $A_{\lambda}(u)=g$. But since $A_{\lambda}{ }^{\prime}(\bar{u})$ is an invertible linear operator, the 
Inverse Function Theorem implies that $A_{\lambda}(u)$ is a local homeomorphism of a neighborhood $U$ of $\bar{u}$ onto a neighborhood of $g$. This contradicts the fact that the set $A_{\lambda}^{-1}(g)$ has infinite cardinality near $\bar{u}$.

Property (iv) follows immediately from the properness of $A_{\lambda}(u)$ (i.e. Theorem 1). For $g \notin A_{\lambda}(S)$ all critical points of $\mathscr{I}_{\lambda}(u)$ are necessarily nondegenerate. Consequently the Morse inequalities of [6] hold, by Theorem 3 , since $\mathscr{I}_{\lambda}(u)$ is bounded below and $\mathscr{I}_{\lambda}(u)$ can have only a finite number of critical points of given Morse index [by virtue of (ii)].

Thus it remains to prove (iii). Let $U$ be an open component of $H-A_{\lambda}(S)$, and let $y \in U$. Setting $N(z)=$ cardinality of $A_{\lambda}^{-1}(z)$, we let $g_{n} \rightarrow g$ for $g_{n} \in U$ and show that $N\left(g_{n}\right)=N(g)$ for $n$ sufficiently large. To this end, observe that by (ii), $N(y)$ is finite, and if the solutions of $A_{\lambda}(u)=g$ occur at $u_{1}, u_{2}, \ldots, u_{k}$, then $\left[\right.$ since $\left.g \notin A_{\lambda}(S)\right]$ the Inverse Function Theorem implies that for $n$ sufficiently large the equation $A_{\lambda}(u)=g_{n}$ has a unique solution $u_{i}^{(n)}$ near $u_{i}$ for each $i(i=1, \ldots, k)$. On the other hand, if $Q\left(u_{i}\right)$ are sufficiently small neighborhoods of $u_{i}$ $(i=1, \ldots, k)$, the equation $A_{\lambda}(u)=g$ will have no solutions on $U_{k}=U$ $-\bigcup_{i=1}^{k} Q\left(u_{i}\right)$. Thus the slightly perturbed equation, $A_{\lambda}(u)-\left(g_{n}-g\right)=g$, will also have no solutions on $U_{k}$ [i.e. $A_{\lambda}(u)=g_{n}$ will have no solutions on $U_{k}$ ]. Hence $N(y)$ is continuous at all points of $U$. Since $N(y)$ is also integer-valued, $N(y)$ must be constant on $U$, as required.

\section{Section III. Global Properties of Equilibrium States}

Here we study the properties of the solutions of the system $(1 \mathrm{a}-\mathrm{d})$ as a function of the real numbers $\lambda$ and $\|g\|$, by using the results of Section II. It is very useful to relate the solutions of (2) to the spectrum of the operator $L$ defined in Lemma A. Indeed, we denote by $\left\{\lambda_{j}\right\}$ the real numbers $\lambda_{j}$ at which the linear operator $I-\lambda L$ is not invertible. By known results of linear self-adjoint spectral theory [5], the compactness of $L$ implies that $\left|\lambda_{j}\right| \rightarrow \infty$ as $j \rightarrow \infty$, and for example, if the force acting on the edge of the plate is purely compressive, we may suppose that $\lambda_{j}>0$. In general we order the numbers $\left\{\lambda_{j}\right\}$ in such a way that (i) $\left\{\lambda_{j}\right\}=\left\{\lambda_{j}{ }^{+}\right\} \cup\left\{\lambda_{j}{ }^{-}\right\}$with $\cdots \leqq \lambda_{2}{ }^{-} \leqq \lambda_{1}{ }^{-} \leqq 0<\lambda_{1}{ }^{+} \leqq \lambda_{2}{ }^{+} \leqq \lambda_{3}{ }^{+} \leqq \cdots$.

The first general result we prove is

Theorem 5. For any values of $\lambda$ and $g$ :

(i) The system $(1 \mathrm{a}-\mathrm{d})$ always possesses a solution $(\bar{u}, \bar{f})$ that renders the functional $\mathscr{I}_{\lambda}(u)$ an absolute minimum;

(ii) Any solution will satisfy the a priori bound (7);

(iii) For fixed $\lambda<\lambda_{1}$ and $\|g\|$ accordingly small (but nonzero) the minimizing solution of (i) is unique;

(iv) If the system admits 2 isolated absolute or relative minima, then the system will always possess a third distinct solution. 
Proof. (i) There are various proofs possible for this result. Perhaps the simplest is the observation that clearly the function $\mathscr{I}_{\lambda}(u)$ is lower semi-continuous with respect to weak convergence in $H$. (This follows immediately for [7, pp. 110-112].) By Theorem 3, $\inf _{H} \mathscr{I}_{\lambda}(u)=\alpha>-\infty$. Consequently by Theorem 3 again, any minimizing sequence $\left\{u_{n}\right\}$ in $H$ has a weakly convergent subsequence, which we again denote by $\left\{u_{n}\right\}$, with weak limit $\bar{u}$. Thus $\mathscr{I}_{\lambda}(\bar{u})=\alpha$ and $\bar{u}$ is the desired solution of (2) since $\mathscr{I}_{\lambda}(u)$ is differentiable.

(ii) This is an immediate consequence of the Corollary of Section II.

(iii) If $\lambda<\lambda_{1}$, and $\bar{\omega}$ satisfies (2), the Inverse Function Theorem applied to the operator $f(\omega)=\omega+C \omega-\lambda L \omega$ implies that $f$ is a local homeomorphism near $\omega=0$, since $f^{\prime}(0)=I-\lambda L$ is invertible. Thus, since $f(0)=0$ for $\|g\|$ sufficiently small, (2) has a unique solution $\bar{u}$ near $\omega=0$. On the other hand, taking the inner product of (2) with $\bar{\omega}$, we find that since $((I-\lambda L) \omega, \omega) \geqq k\|\omega\|^{2}$,

$$
k_{\lambda}\|\omega\|^{2} \leqq\|g\|\|\omega\| .
$$

Thus $\|\omega\| \leqq k_{\lambda}{ }^{-1}\|g\|$, and consequently choosing $\|g\|$ sufficiently small (but positive relative to $k_{\lambda}$ ), any solution of (2) must lie in a domain of uniqueness for $f$.

(iv) To establish the existence of a third critical point, we observe that by Theorem 4 (iv), $\mathscr{I}_{\lambda}(u)$ satisfies the Palais-Smale Condition C on $H$. On the other hand, Theorem 4 implies that $\mathscr{I}_{\lambda}(u)$ is bounded below on $H$. Thus (iv) follows by [8, p. 396], if the given absolute minima are nondegenerate critical points. In the general case the results follow from relatively standard arguments, as in [9], or [7, pp. 59-62].

For "generic" values of $g$ the following holds.

Theorem 6. For fixed $\lambda$, and almost all values of $g$ (i.e. $g \notin A_{\lambda}(S)$ ), the number of solutions of $(1 \mathrm{a}-\mathrm{d})$ will be a finite integer. Moreover, for such values of $g$, the following Morse inequalities hold for these solutions. Let $\mathscr{M}_{i}$ be the number of critical points of Morse index $i$ for $\mathscr{I}_{\lambda}(u)$, then

$$
\begin{aligned}
\mathscr{M}_{0} & \geqq 1 \\
\mathscr{M}_{1}-\mathscr{M}_{0} & \geqq-1 \\
\mathscr{M}_{2}-\mathscr{M}_{1}+\mathscr{M}_{0} & \geqq 1 \\
\sum_{m=0}^{k}(-1)^{k-m} \mathscr{M}_{m} & \geqq(-1)^{k} \\
\sum_{m=0}^{\infty}(-1)^{m} \mathscr{M}_{m} & =1 .
\end{aligned}
$$

Proof. If $g \notin A_{\lambda}(S)$, there can be at most a finite number of solutions for (2), since the mapping $A_{\lambda}(u)$ is proper (Theorem 1) and the a priori bound of the Corollary holds. Indeed, otherwise some neighborhood 
of a solution $\bar{u}$ would contain an infinite number of solutions so that $g \in A_{\lambda}(S)$, contrary to hypothesis. The validity of the Morse inequalities follows immediately from [6], since the functional $\mathscr{I}_{\lambda}(u)$ is bounded from below (Theorem 4) and satisfies the Palais-Smale Condition $\mathrm{C}$ on $H$. Indeed, the relevant Betti numbers $R_{i}$ of $\mathscr{M}^{b}=\left\{u \mid \mathscr{I}_{\lambda}(u) \leqq b\right\}$ (for $b$ chosen so large that all critical points of $\mathscr{I}_{\lambda}(u)$ lie in $\left.\mathscr{M}^{b}\right)$ are one if $i=0$ and zero otherwise.

Remark. Even if $g \in A(S)$, it is conjectured that a slight perturbation of $\Omega$ will cause $g$ to be a nonsingular value for the slightly perturbed domain.

We now give a somewhat more precise result in case $g=0$, that does not require that $0 \in\left(H-A_{\lambda}(S)\right)$.

Theorem 7. For $\lambda \in\left(\lambda_{n}{ }^{+}, \lambda_{n+1}{ }^{+}\right]$or $\lambda \in\left(\lambda_{n}{ }^{-}, \lambda_{n+1}{ }^{-}\right]$, and $g=0$ the system $(1 \mathrm{a}-\mathrm{d})$ has at least $n$ pairs of distinct nontrivial solutions $\left( \pm u_{n}(\lambda)\right)$. These solutions are characterized by the variational principle (11) defined below.

Proof. We consider the functional $\mathscr{I}_{\lambda}(u)$ with $g=0$ defined over $H$. For either $\lambda>\lambda_{1}{ }^{+}$or $\lambda<\lambda_{1}{ }^{-}$

$$
c_{0}=\inf _{u \in H} \mathscr{I}_{\lambda}(u)<0
$$

Indeed, if $u_{1}$ is a normalized eigenvector associated with $\lambda_{1}{ }^{+}$or $\lambda_{1}{ }^{-}$, then for sufficiently small positive $c$,

$$
c_{0}<\mathscr{I}_{\lambda}\left(c u_{1}\right)=c^{2}\left(1-\frac{\lambda}{\lambda_{1}}\right)\left\|u_{1}\right\|^{2}+\frac{1}{2} c^{4}\left(C u_{1}, u_{1}\right)<0 .
$$

Thus (by Theorem 5), $c_{0}$ is a critical value corresponding to nontrivial (i.e. $\left.u_{1}(\lambda) \neq 0\right)$ critical points $\pm u_{1}(\lambda)$. For larger values of $|\lambda|$, topological generalizations of the critical point $c_{0}$ seem to be required to obtain further solutions.

Thus we shall follow an argument due to Clark [10]. Let the class of closed sets of $\Sigma=H-\{0\}$ symmetric about the origin be denoted by $S(\Sigma)$. Then, the genus of $A \in S(\Sigma), g(A)=1+\operatorname{cat}(\tilde{A}, \tilde{\Sigma})$, where $\operatorname{cat}\left(\tilde{A}, \Sigma / Z_{2}\right)$ denotes the Ljusternik-Schnirelmann category of the $\tilde{A}(A$ with antipodal points identified) relative to $\tilde{\Sigma}$ ( $\Sigma$ with antipodal points identified). Then the arguments of [10] together with Theorem 4 show that the numbers

$$
c_{n}=c_{n}\left(\mathscr{I}_{\lambda}(u)\right)=\inf _{g(A) \geqq n+1} \sup _{u \in A} \mathscr{I}_{\lambda}(u) \quad(n=0,1,2, \ldots),
$$

if finite and strictly negative, are critical values for $\mathscr{I}_{\lambda}(u)$; and that the associated critical points on $\tilde{\Sigma}$ can be chosen as distinct. Clearly by 
Theorem 3, and (10), $-\infty<c_{0} \leqq c_{1} \leqq c_{2} \leqq \cdots$. Thus to establish the desired result we need only show that, for $\lambda \in\left(\lambda_{n}{ }^{+}, \lambda_{n+1}{ }^{+}\right]$or $\left(\lambda_{n}{ }^{-}, \lambda_{n+1}{ }^{-}\right]$, the numbers $c_{1}, c_{2}, \ldots, c_{n-1}$ are strictly negative. To this end, we argue as in [10]. Let $A_{n}{ }^{ \pm}$be the closed linear subspace formed from the eigenvectors $\left(u_{1}{ }^{ \pm}, u_{2}{ }^{ \pm}, \ldots, u_{n}{ }^{ \pm}\right)$of $L$ (with zero deleted), and let $S_{n}{ }^{c}$ denote the sphere of radius $c$ in $A_{n}{ }^{+}$or $A_{n}{ }^{-}$. Then $g\left(S_{n}{ }^{c}\right)=n+1$, and for sufficiently small $c$ and normalized $\bar{u} \in S_{n}$, there is a constant $\beta(\lambda)<0$ such that $((I-\lambda L) \bar{u}, \bar{u})<\beta(\lambda)\|\bar{u}\|^{2}$. Hence

$$
\begin{aligned}
c_{n}<\mathscr{I}_{\lambda}(c \bar{u})= & \sup _{\|u\|=1}\left\{-c^{2} \beta(\lambda)\|\bar{u}\|^{2}+\frac{1}{2} c^{4}(C \bar{u}, \bar{u})\right\} \\
& <0 \quad \text { (as required) } .
\end{aligned}
$$

Consequently, the desired result is established.

An Example. Consider a thin circular clamped plate acted on by a uniform compressive force of magnitude $\lambda$ along its edge. As is wellknown, for $\lambda \leqq \lambda_{1}$ (the lowest eigenvalue of the associated linearized problem) the plate does not deform out of its plane, while for $\lambda>\lambda_{1}$ the plate does "buckle". For $\lambda$ slightly larger than $\lambda_{1}$ this deformation is observed to be radially symmetric. However as $\lambda$ is increased still further the non-planar radially symmetric equilibrium state (while continuing to exist) itself loses stability to a new asymmetric equilibrium state. This situation is discussed in Yanowitch [12], and demonstrates the physical importance of the multiple equilibrium states shown to exist in Theorem 7. Indeed, it seems likely that the critical points found in Theorem 7 are the "continuations" of the solutions bifurcating from the eigenvalues $\lambda_{n}$ of the linearized problem (about the planar equilibrium state).

Similarly if the plate is subjected to a uniformly distributed normal force of magnitude $\mu$ (but no edge forces), then it is again shown in [12] (for suitable edge conditions) that for sufficiently small $\mu$, the stable deformation will be radially symmetric. On the other hand, as $\mu$ increases, while continuing to exist, the unique radially symmetric equilibrium state loses stability to an asymmetric equilibrium state. (Here stable means that the second variation of $\mathscr{I}_{\lambda}(u)$ is positive definite at the given equilibrium state.)

Corollary. Suppose $0 \in\left(H-A_{\lambda}(S)\right)$, then the conclusions of Theorem 7 hold also for nonzero $g$, provided $\|g\|$ is sufficiently small.

Proof. If $0 \notin A_{\lambda}(S)$, then by Theorem 4 (iii), the number of solutions of the equation $A_{\lambda}(u)=g$ is constant in the component $C$ of $H-A_{\lambda}(S)$ containing 0 . Since $C$ contains an open ball about 0 , the Corollary follows provided $g$ is contained in this ball. 
Remark. In conclusion we note that the results of $[1,2]$ explored the structure of the equilibrium states $(w, f)$ of $(1 \mathrm{a}-\mathrm{d})$ with $g=0$ and with fixed norm, but without regard to $\lambda$. In contrast, here, we have fixed $\lambda$ and $g$ and investigated the solutions $(w, f)$ of $(1 \mathrm{a}-\mathrm{d})$ without regard to their norm. The exact relationship between these approaches seems difficult to determine. We conjecture that if $g=0$ the solutions $\left(w_{\lambda}, f_{\lambda}, \lambda\right)$ bifurcating from $\left(0,0, \lambda_{n}^{ \pm}\right)$define smooth curves with $\left\|w_{\lambda}\right\|^{2}+\left\|f_{\lambda}\right\|^{2} \rightarrow \infty$ as $|\lambda| \rightarrow \infty$. Such behavior is consistent with all the facts so far known about the solutions of the von Karman equations, and would explain the relationship between the two approaches to the study of equilibrium states.

\section{References}

1. Berger, M.: On von Karman's equations and the buckling of a thin elastic plate I. Commun. Pure Appl. Math. 20, 687-720 (1967)

2. Berger, M., Fife,P.: On von Karman's equations II. Commun. Pure Appl. Math. 21, 227-241 (1968)

3. Knightly, G., Sather, D.: Arch. Rat. Mech. Appl. 36, 65-78 (1970)

4. Krasnoselski, M.: Topological methods for nonlinear integral equations. New York: Pergamon 1956

5. Dieudonné,J.: Elements of analysis, Vol. 1. New York: Academic Press, 1961

6. Palais, R.: Morse theory on Hilbert manifold. Topology 2, 299-240 (1963)

7. Berger, M.S., Berger, M.S.: Perspectives in nonlinearity. New York: Benjamin 1968

8. Smale, S.: Morse theory and a nonlinear generalization of the Dirichlet problem. Ann. Math. 80, 382-396 (1964)

9. Berger, M.: Lectures on nonlinear problems in mathematical analysis (to appear).

10. Clark, D. : A variant of the Ljusternik-Schnirelmann theory. Indiana Math. J. 22, 65-74 (1972)

11. Smale, S.: An infinite dimensional version of Sard's theorem. Am. J. Math. 87, 861-866 (1965)

12. Yanowitch,M.: Nonlinear Buckling of thin elastic plates. Commun. Pure Appl. Math. 9, 661—672 (1956)

Communicated by D. Ruelle

Melvyn S. Berger

Belfer Graduate School of Science

Yeshiva University

Amsterdam Avenue and 186 th Street

New York, N.Y. 10033 